\section{PCMOS - Protein Crystallization Microfluidic Systems}

\section{Unique features of PCMOS}

MEMS (Microelectro-mechanical systems) and Microfluidics-based protein crystallization devices are applicable to highthroughput and high-quality protein crystallization. The high-throughput crystallization devices enable automated preparation and evaluation of a small amount of sample droplet, and active control of several crystallization conditions is possible in high-quality crystallization devices.

Recent progress in macromolecular crystallography requires new methodological technology for both the purpose of high-throughput and high-quality protein crystallization. PCMOS are the first downsized, integrated and high-performance MEMS (MicroElectro-Mechanical Systems) technology-based devices for such kinds of macromolecular crystallization. Some unique features of the PCMOS include the following.

1. Materials are clean and chemically stable.

2. Consumption is of small amounts of protein and precipitant solutions.

3. The systems are compatible with conventional crystallization well plates and tools.

4. They can be applied to high-throughput crystallization screening.

5. Automated crystallization, cryocooling, and X-ray diffraction data collection is possible.

6. Three-dimensional integration enables simple crystallization work.

\section{Device classification}

Crystallization methods and the corresponding PCMOS device names (* under development) are given below.
Vapor diffusion: VD-MOS.

High-throughput vapor diffusion: HTVDMOS.

Microbatch: MB-MOS*.

Liquid-liquid diffusion: LLD-MOS.

Liquid-gel diffusion: LGD-MOS.

Electric-force assisted: EFA-MOS*.

Membrane protein crystallization: MPCMOS*.

\author{
Akira Sanjoh \\ Protein Wave Corporation \\ Bldg Lab Wing/5F \\ 1-7 Hikaridai \\ Seika-cho \\ Kyoto 619-0237
}

\section{Modular field-flow fractionation and dynamic laser light scattering system for size and molecular weight determination}

The new postnova Avalanche (FFF) and Galaxy (light scattering) characterization systems for size and molecular-weight determination of biological macromolecules and particles offer a wide variety of different applications to scientists in research and industry. The systems can be used combined or as single components. The Avalanche field-flow fractionation system (FFF) is used for size separation of macromolecules and biological nanoparticles in a flow separation channel. The separation can be performed very gentlly and quickly without shear forces affecting the samples or aggregates of the sample. The samples can be collected after the separation for further analysis, as REM, TEM, LS etc. Coupled to the Avalanche FFF, the Galaxy Series of Static and Dynamic Laser Light Scattering systems (SLS, DLS, PCS, QELS) is used to determine the hydrodynmaic radius of the samples and the molecular weight. The DLS can be used also as a batch instrument utilizing a microcuvette for determining the size of the macromolecules and particles.

The web address for postnova analytics is http://www.postnova.com.

Thorsten Klein and Peter F. W. Simon

postnova analytics

Gewerbering 15

86922 Eresing

Germany

\section{Eclipse $F^{(i x)}$ separation system for proteins}

The Eclipse $F^{(m)}$ separation system uses the principles of asymmetrical flow field-flow fractionation (AFFF) to separate particles and molecules from a few nanometres to more than a micrometre in size. It integrates with Wyatt Technology's light-scattering detectors, which determine molar mass and size online following separation. The system is ideal for analysing protein solutions and examining aggregation and association states. Measuring the concentration dependence of the light scattering intensity of protein solutions, the second virial coefficient can be calculated, which is a useful parameter to monitor the solvent quality and solution state of the protein. The measurements can be performed in a fully automated way and with high throughput.

The web address for Wyatt Technology Europe is http://www.wyatt.de.

\section{Wyatt Technology Europe}

In der Steubach 9

D-57614 Woldert

Germany 